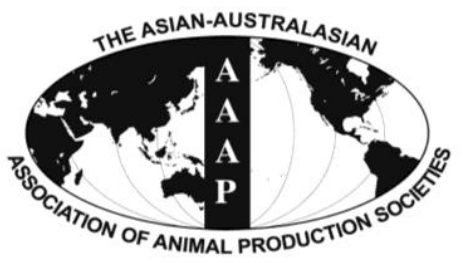

\title{
Physicochemical and Sensory Properties of Red Ginseng Extracts or Red Ginseng Hydrolyzates-added Asiago Cheese during Ripening
}

\author{
Kyung-Hoon Choi, Ji-Young Min, Palanivel Ganesan ${ }^{1}$, In-Hyu Bae ${ }^{2}$, and Hae-Soo Kwak* \\ Department of Food Science and Technology, Sejong University, Seoul 143-747, Korea
}

\begin{abstract}
This study was carried out to investigate physicochemical properties of different concentrations $(0.1 \%, 0.3 \%$, and $0.5 \%)$ of red ginseng hydrolyzates (RGH)- or red ginseng extract (RGE)-added Asiago cheeses (AC) during ripening at $14^{\circ} \mathrm{C}$ for 4 months. The moisture content significantly increased with increasing concentrations of both RGH- and RGE- added AC ( $<<0.05)$. While RGHAC and RGEAC were more yellow and darker with increasing concentrations than that of control $(\mathrm{p}<0.05)$, the color was not influenced from the hydrolysis. In texture analysis, hardness, cohesiveness, and chewiness of RGHAC and RGEAC significantly decreased compared to the control during the ripening $(\mathrm{p}<0.05)$. In sensory analysis, bitterness and ginseng flavor and taste scores increased significantly with increasing the concentrations of RGH and RGE during ripening $(\mathrm{p}<0.05)$. In conclusion, the addition of RGH and RGE into cheese slightly influenced the properties of Asiago cheese, and similarities were observed between RGHAC and RGEAC. Thus, the lower concentrations $(0.1 \%$ to $0.3 \%)$ of RGH and RGE added to AC were preferred for color, texture, and sensory during the ripening, therefore, these cheeses would be worth developing commercially. (Key Words: Red Ginseng Hydrolyzates, Asiago Cheese, Physicochemical Property)
\end{abstract}

\section{INTRODUCTION}

Korean ginseng is well known for its various bioactive functions, such as antidiabetic, anti-mutagenic, antiinflammatory, antioxidant, and anticancer (Lee et al., 1997; Chung et al., 2001; Kim et al., 2005; Kim et al., 2006). They are available in two forms in the commercial market based on the processing types involved, known as white and red ginsengs. White ginseng is obtained from peeling the fresh ginseng and air drying, whereas red ginseng is obtained from steaming ginseng at $98^{\circ} \mathrm{C}$ to $100^{\circ} \mathrm{C}$ without peeling (Lee et al., 2012). Steaming process enhances the richness of many functional compounds, such as $\operatorname{Rg} 3, \operatorname{Rg} 5$, and $\mathrm{Rk} 1$ ginsenosides, which are mostly absent from white ginseng (Kwon et al., 2001). Most of the functional

\footnotetext{
* Corresponding Author: H. S. Kwak. Tel: +82-2-3408-3226, Fax: +82-2-3408-4319, E-mail: kwakhs@ sejong.ac.kr

${ }^{1}$ Department of Food Technology, Universiti Putra Malaysia, Serdang43400, Malaysia.

2 Department of Animal Science and Technology, Sunchon National University, Sunchon 540-950, Korea.

Submitted Jan. 22, 2014; Revised Mar. 20, 2014; Accepted Apr. 8, 2014
}

ginsenosides are readily metabolized by the intestinal bacteria and are easily absorbed into the blood stream to act as functional bioactive compounds.

Recent studies have suggested that enzyme-hydrolysis is an effective way to increase the oral bioavailability of the bioactive compound. Ginsenoside Rb1 is the main component in ginsenosides. It is a protopanaxadiol-type ginsenoside that has a structure similar to that of compound K (20-O- $\beta$-D-glucopyranosyl-20(S)-protopanaxadiol, C-K) (Bae et al., 2002; Paek et al., 2006). By enzymatically hydrolyzing, the two glucose molecules at $\mathrm{C}-3$ and one of the glucose molecules at $\mathrm{C}-20$, ginsenosides $\mathrm{Rb} 1$ can easily be transformed to $\mathrm{C}-\mathrm{K}$. The $\mathrm{C}-\mathrm{K}$ does not exist in natural products and the natural transforming ability of human intestinal bacteria is rather limited, therefore, much attention ha $3 \mathrm{~s}$ been paid to the preparation of C-K (Akao et al., 1998). However, none of the methods were suitable for preparation of $\mathrm{C}-\mathrm{K}$, because both glycone moieties at $\mathrm{C}-20$ could be cleaved nonspecifically under chemical hydrolysis conditions.

Cheese is one of the most nutritious dairy foods which provides a rich source of protein, fat, and minerals (Jung et 
al., 2013). Among the cheeses, Asiago cheese (AC) is an Italian type hard cheese widely consumed in many European and oriental countries. Based on the ageing, it is classified into two forms; "Pressato" with a lower ageing period and "Asiago d'Allevo" with an ageing period of 6 months. The typical characteristics of the AC is that it does not undergo any flavor change after an ageing period of 9 months. Besides the higher nutritional content of $\mathrm{AC}$, today's health conscious society requires value-added functional AC. Some researchers also reported adding functional compounds to a hard cheese, such as evening primrose oil into Cheddar cheese, to lower blood cholesterol (Kim et al., 2006) and the antioxidant effect of ginseng in yogurt (Kim et al., 2008). To our knowledge, there is no report of adding ginseng or its hydrolyzate to AC. Therefore, the objective of this work was focused on the physiochemical and sensory properties of cheese with added red ginseng extracts (RGE) or its hydrolyzates.

\section{MATERIALS AND METHODS}

\section{Materials}

Red ginseng hydrolyzates (RGH) and RGE were provided from Dongwon F\&B Co., Ltd. (Seongnam, Korea). The contents of ginsenosides in RGE and RGH were shown in Table 1.

\section{Manufacture of Asiago cheese}

Asiago cheese was produced using the method described by Gazzetta (2006). Raw milk (100 kg) was pasteurized at $65^{\circ} \mathrm{C}$ for $30 \mathrm{~min}$ and it was cooled to $35^{\circ} \mathrm{C}$ and then $1 \%$ of a freeze-dried direct vat set mesophilic aromatic lactic acid starter culture (FLORA DANICA, Chr. Hansen's Lab., Copenhagen, Denmark) and $0.02 \% \mathrm{CaCl}_{2}$ were added to the milk. After $50 \mathrm{~min}, 0.02 \%(19 \mathrm{~mL})$ of rennet (diluted 1:20) (Chr. Hansen's Lab., Copenhagen, Denmark) was added to milk and allowed to stand for 30 min. After coagulating, curds were cut, set for $3 \mathrm{~min}$, gradually heated from 35 to $40^{\circ} \mathrm{C}$ for $20 \mathrm{~min}$ and stirred at $40^{\circ} \mathrm{C}$ for $20 \mathrm{~min}$ (1st heating). The 2 nd heating proceeded from $40^{\circ} \mathrm{C}$ to $45^{\circ} \mathrm{C}$ for 20 with stirring at $45^{\circ} \mathrm{C}$ for $30 \mathrm{~min}$. Whey was totally removed for 20 min during setting and rubbed for removing residual whey until it disappeared. Different concentrations $(0.1 \%, 0.3 \%$, and $0.5 \%)$ of RGE and RGH were evenly added to curds before they were put

Table 1. Contents of ginsenosides in red ginseng hydrolyzates (RGH) and red ginseng extracts (RGE)

\begin{tabular}{lcc}
\hline Ginsenoside & RGH $(\mathrm{mg} / \mathrm{g})$ & RGE $(\mathrm{mg} / \mathrm{g})$ \\
\hline $\mathrm{Rg} 1+\mathrm{Rb} 1$ & 15 & 15 \\
$\mathrm{Rh} 1+\mathrm{Rg} 3$ & 15 & 15 \\
$\mathrm{CK}+\mathrm{F} 2$ & 2 & - \\
\hline
\end{tabular}

CK, compound K (20-O- $\beta$-D-glucopyranosyl-20(S)-protopanaxadiol). into molds for pressing. After that, the curds were pressed at 6 times of the curd weight overnight. After pressing, the fresh cheeses were brined in $20 \%$ brine solution ( $\mathrm{pH} 5.2$ to 5.4) for $2 \mathrm{~h}$. After draining-off from the brine and drying the cheese, they were ripened at $14^{\circ} \mathrm{C}$ for 6 months with a relative humidity of $85 \%$. Samples for analysis were periodically taken at $0,1,3,4,5$, and 6 months of ripening

\section{Proximate analysis}

Asiago cheese samples were analyzed for moisture, protein, and fat according to the methods of AOAC (AOAC, 2000).

\section{Lactic acid bacteria count}

De Man, Rogosa and Sharpe broth (DifcoLaboratores, Detroit, MN, USA) and $0.004 \%$ bromophenol blue was used for lactic acid bacteria (LAB) counts. One gram of cheese sample was dispersed in $9 \mathrm{~mL}$ of $2 \%$ sodium citrate and it was prepared by homogenizing for $1 \mathrm{~min}$ at 1,500 $\mathrm{rpm}$. One milliliter of prepared sample was diluted with 9 $\mathrm{mL}$ of sterile $0.8 \% \mathrm{NaCl}$. Subsequent dilutions of each sample were plated in triplicate and incubated at $37^{\circ} \mathrm{C}$ for $72 \mathrm{~h}$.

\section{Color measurement}

Color values were compared between the control and sample cheeses using a colorimeter (Minolta CT-310, Tokyo, Japan) after calibrating its original value with a standard plate. Measured $\mathrm{L}^{*}, \mathrm{a}^{*}$, and $\mathrm{b}^{*}$ values were used as indicators of lightness, redness, and yellowness, respectively. All samples were measured in triplicate.

\section{Texture profile analysis}

Cube-shaped samples $\left(1.5 \mathrm{~cm}^{3}\right)$ of each cheese at each stage of ripening were cut from the central part of the cheese. Textural properties, such as hardness, cohesiveness, springiness, gumminess, and chewiness of the cheese, were measured with a TMS-Pro Texture Analyzer (Food Technology Co., Sterling, VA, USA) according to the modified method of Kanawjia et al. (1995).

\section{Sensory analysis}

For the sensory test, red ginseng-treated $\mathrm{AC}$ were ripened at $14^{\circ} \mathrm{C}$ for $0,1,2,3$, and 4 months with relative humidity of $85 \%$. A 10 -member trained panel evaluated randomly coded AC. The appearance, texture, color, flavor, and taste were evaluated on a 7-point scale as follows; $(1=$ very weak, $4=$ moderate, $7=$ very strong). A randomized, balanced, complete block design was used by Cochran and Cox (1957).

\section{Statistical analysis}

The results are expressed as mean \pm SD. Statistical 
Table 2. Proximate analysis in red ginseng hydrolyzate-added Asiago cheese (\%)

\begin{tabular}{lcccc}
\hline Concentration of sample $(\%, w t / w t)$ & Moisture & Fat & Protein & Ash \\
\hline Control & $34.35 \pm 0.27^{\mathrm{d} 4)}$ & $33.54 \pm 0.27^{\mathrm{a}}$ & $21.53 \pm 0.19^{\mathrm{a}}$ & $10.88 \pm 0.12^{\mathrm{b}}$ \\
RGEAC $(0.1)$ & $35.54 \pm 0.31^{\mathrm{bc}}$ & $33.40 \pm 0.26^{\mathrm{a}}$ & $21.34 \pm 0.34^{\mathrm{a}}$ & $10.11 \pm 0.25^{\mathrm{b}}$ \\
RGEAC $(0.3)$ & $36.05 \pm 0.67^{\mathrm{ab}}$ & $33.30 \pm 0.87^{\mathrm{a}}$ & $20.00 \pm 0.16^{\mathrm{ab}}$ & $10.26 \pm 0.30^{\mathrm{ab}}$ \\
RGEAC $(0.5)$ & $36.64 \pm 0.10^{\mathrm{a}}$ & $32.97 \pm 0.14^{\mathrm{b}}$ & $19.79 \pm 0.50^{\mathrm{b}}$ & $10.77 \pm 0.14^{\mathrm{ab}}$ \\
RGHAC $(0.1)$ & $35.26 \pm 0.34^{\mathrm{c}}$ & $33.10 \pm 0.51^{\mathrm{ab}}$ & $21.11 \pm 0.11^{\mathrm{a}}$ & $10.78 \pm 0.18^{\mathrm{ab}}$ \\
RGHAC $(0.3)$ & $35.66 \pm 0.15^{\mathrm{bc}}$ & $32.78 \pm 0.64^{\mathrm{b}}$ & $20.04 \pm 0.40^{\mathrm{ab}}$ & $10.49 \pm 0.24^{\mathrm{ab}}$ \\
RGHAC $(0.5)$ & $36.14 \pm 0.62^{\mathrm{ab}}$ & $32.55 \pm 0.11^{\mathrm{b}}$ & $19.88 \pm 0.08^{\mathrm{b}}$ & $11.18 \pm 0.42^{\mathrm{a}}$ \\
\hline
\end{tabular}

Control, Asiago cheese; RGEAC, red ginseng extract-added Asiago cheese; RGHAC, red ginseng hydrolyzate-added Asiago cheese; SD, standard deviation.

Data values were expressed as mean $\pm \mathrm{SD}(\mathrm{n}=3)$. Values within the same column with different superscripts are significantly different at $\mathrm{p}<0.05$ by Duncan's multiple range test.

significance was determined by analysis of variance and subsequent Duncan's multiple range test $(\mathrm{p}<0.05)$. The analysis was performed using SAS statistical software.

\section{RESULTS AND DISCUSSION}

\section{Proximate analysis}

The composition of AC added with different concentrations $(0.1 \%, 0.3 \%$, and $0.5 \%)$ of RGH or RGE at 0 month are presented in Table 2. Moisture contents of red ginseng extract-added Asiago cheese (RGEAC) and red ginseng hydrolyzates-added Asiago cheese (RGHAC) were significantly higher than that in control, and increasing the concentrations of RGE and RGH in cheeses also significantly increased moisture contents $(\mathrm{p}<0.05)$. Fat and protein contents were relatively decreased by adding RGE and RGH. While ash was increased with increasing concentrations of RGE and RGH in cheese, the highest ash content was observed in $\operatorname{RGHAC}(0.5 \%$, wt/wt). High amounts of moisture in RGEAC and RGHAC were influenced by the soluble content obtained from ethanol extraction, but enzyme hydrolysis that produced smaller molecules did not affect the moisture composition. Ethanol extraction of red ginseng contained mainly glycosides that may not have influenced the quantitative composition of the ginseng.

\section{Lactic acid bacteria count}

The changes in LAB counts of RGHAC and RGEAC ripened at $14^{\circ} \mathrm{C}$ for 4 months are shown in Table 3 . Increasing the ripening periods caused the LAB counts to be significantly decreased in both cheeses $(p<0.05)$, but LAB count was not influenced by the addition of RGH or RGE in the cheeses. Over the ripening period, LAB in $0.5 \%$ RGHAC and $0.5 \%$ RGEAC decreased from $2.90 \times 10^{9}$ to $1.95 \times 10^{7}$ colony-forming unit $(\mathrm{CFU}) / \mathrm{mL}$ and from $2.90 \times 10^{9}$ to $1.95 \times 10^{7} \mathrm{CFU} / \mathrm{mL}$, respectively. Similarly, Kim et al. (2008) also reported that RGE addition to yogurt does not effect the LAB counts in the yogurt. It is further confirmed in our study, even the addition of the RGH rich in glycosides, such as $\mathrm{Rg} 1, \mathrm{Rb} 1, \mathrm{Rh} 1$, and $\mathrm{Rg} 3$ has no adverse effect on the LAB counts.

\section{Color}

The changes in color of different concentrations $(0.1 \%$, $0.3 \%$, and $0.5 \%$ ) of RGH-or RGE-added AC samples ripened at $14^{\circ} \mathrm{C}$ for 4 months are presented in Table 4 . The $\mathrm{L}^{*}$ values decreased significantly with increasing ripening periods over 4 months, and the concentrations of RGH or RGE in cheeses also significantly decreased the $L^{*}$ value

Table 3. Change of lactic acid bacteria count in red ginseng hydrozate- or red ginseng extracts-added Asiago cheese ripened at $14^{\circ} \mathrm{C}$ for 4 months $(\mathrm{CFU} / \mathrm{mL})$

\begin{tabular}{|c|c|c|c|c|c|}
\hline \multirow{2}{*}{$\begin{array}{l}\text { Concentration of } \\
\text { sample }(\%, w t / w t)\end{array}$} & \multicolumn{5}{|c|}{ Ripening period (month) } \\
\hline & $0^{1}$ & 1 & 2 & 3 & 4 \\
\hline Control & $3.15 \pm 0.56 \times 10^{9 \mathrm{aA}}$ & $7.91 \pm 0.11 \times 10^{8 \mathrm{bA}}$ & $1.45 \pm 0.02 \times 10^{8 \mathrm{cAB}}$ & $6.90 \pm 0.13 \times 10^{7 \mathrm{dA}}$ & $1.95 \pm 0.63 \times 10^{7 \mathrm{eA}}$ \\
\hline RGEAC (0.1) & $2.90 \pm 0.26 \times 10^{9 \mathrm{aAB}}$ & $7.89 \pm 0.25 \times 10^{8 \mathrm{bAB}}$ & $1.59 \pm 0.17 \times 10^{8 \mathrm{cA}}$ & $6.93 \pm 0.51 \times 10^{7 \mathrm{dA}}$ & $1.99 \pm 0.52 \times 10^{7 \mathrm{eA}}$ \\
\hline RGEAC (0.3) & $2.85 \pm 0.91 \times 10^{9 \mathrm{aBC}}$ & $7.90 \pm 0.37 \times 10^{8 \mathrm{bAB}}$ & $1.31 \pm 0.21 \times 10^{8 \mathrm{cB}}$ & $6.81 \pm 0.45 \times 10^{7 \mathrm{dA}}$ & $1.91 \pm 0.24 \times 10^{7 \mathrm{eB}}$ \\
\hline RGEAC (0.5) & $2.93 \pm 0.12 \times 10^{9 \mathrm{aAB}}$ & $8.05 \pm 0.22 \times 10^{8 \mathrm{bA}}$ & $1.45 \pm 0.35 \times 10^{8 \mathrm{cAB}}$ & $6.95 \pm 0.7 \times 10^{7 \mathrm{dA}}$ & $1.95 \pm 0.28 \times 10^{7 \mathrm{eAB}}$ \\
\hline RGHAC (0.1) & $2.91 \pm 0.33 \times 10^{9 \mathrm{aAB}}$ & $7.95 \pm 0.74 \times 10^{8 \mathrm{bA}}$ & $1.49 \pm 0.27 \times 10^{8 \mathrm{cA}}$ & $6.79 \pm 0.31 \times 10^{7 \mathrm{dAB}}$ & $1.99 \pm 0.73 \times 10^{7 \mathrm{eA}}$ \\
\hline RGHAC (0.3) & $2.95 \pm 0.37 \times 10^{9 \mathrm{aAB}}$ & $7.91 \pm 0.10 \times 10^{8 \mathrm{bA}}$ & $1.51 \pm 0.46 \times 10^{8 \mathrm{cA}}$ & $6.96 \pm 0.54 \times 10^{7 \mathrm{dAB}}$ & $1.91 \pm 0.30 \times 10^{7 \mathrm{eB}}$ \\
\hline RGHAC (0.5) & $2.90 \pm 0.71 \times 10^{9 \mathrm{aAB}}$ & $7.75 \pm 0.61 \times 10^{8 \mathrm{bB}}$ & $1.55 \pm 0.44 \times 10^{8 \mathrm{cA}}$ & $6.45 \pm 0.18 \times 10^{7 \mathrm{~dB}}$ & $1.95 \pm 0.09 \times 10^{7 \mathrm{eA}}$ \\
\hline
\end{tabular}

CFU, colony-forming unit; Control, Asiago cheese; RGEAC, red ginseng extract-added Asiago cheese; RGHAC, red ginseng hydrolyzate-added Asiago cheese; SD, standard deviation.

${ }^{1} 0$ month means the sample obtained after 48 hours from cheese making.

Values are mean \pm SD $(n=10)$. Means with different superscripts in a row (a-e) and column $(A-G)$ are significant at $\mathrm{p}<0.05$ by Ducan's multiple range test. 
Table 4. Changes in color of red ginseng hydrolyzate- or red ginseng extracts-added Asiago cheese ripened at $14^{\circ} \mathrm{C}$ for 4 months

\begin{tabular}{|c|c|c|c|c|c|c|}
\hline \multirow{2}{*}{ Color value } & \multirow{2}{*}{$\begin{array}{c}\text { Concentration of } \\
\text { sample }(\%, w t / w t)\end{array}$} & \multicolumn{5}{|c|}{ Ripening period (month) } \\
\hline & & $0^{1}$ & 1 & 2 & 3 & 4 \\
\hline \multirow[t]{7}{*}{$\mathrm{L}^{*}$} & Control & $80.57 \pm 0.6^{\mathrm{aA}}$ & $79.84 \pm 0.67^{\mathrm{bA}}$ & $77.14 \pm 0.73^{\mathrm{cA}}$ & $75.84 \pm 0.31^{\mathrm{dA}}$ & $75.15 \pm 0.37^{\mathrm{eA}}$ \\
\hline & RGEAC (0.1) & $80.63 \pm 0.67^{\mathrm{aA}}$ & $79.14 \pm 0.47^{\mathrm{bB}}$ & $77.42 \pm 0.76^{\mathrm{cA}}$ & $76.16 \pm 0.37^{\mathrm{dA}}$ & $75.45 \pm 0.29^{\mathrm{eA}}$ \\
\hline & RGEAC (0.3) & $79.70 \pm 0.23^{\mathrm{aB}}$ & $76.57 \pm 0.94^{\mathrm{bD}}$ & $76.01 \pm 0.76^{\mathrm{cB}}$ & $74.49 \pm 0.32^{\mathrm{dC}}$ & $74.31 \pm 0.35^{\mathrm{dB}}$ \\
\hline & RGEAC (0.5) & $78.53 \pm 0.28^{\mathrm{aD}}$ & $73.71 \pm 0.66^{\mathrm{bE}}$ & $74.20 \pm 0.72^{\mathrm{cD}}$ & $73.65 \pm 0.34^{\mathrm{cD}}$ & $71.95 \pm 0.52^{\mathrm{dD}}$ \\
\hline & RGHAC $(0.1)$ & $80.67 \pm 0.28^{\mathrm{aA}}$ & $78.02 \pm 1.17^{\mathrm{bC}}$ & $77.22 \pm 0.69^{\mathrm{cA}}$ & $76.15 \pm 0.66^{\mathrm{dA}}$ & $75.29 \pm 0.21^{\mathrm{eA}}$ \\
\hline & RGHAC (0.3) & $79.24 \pm 0.14^{\mathrm{aC}}$ & $76.10 \pm 0.32^{\mathrm{bD}}$ & $75.24 \pm 0.6^{\mathrm{cC}}$ & $75.00 \pm 0.46^{\mathrm{cB}}$ & $73.31 \pm 0.35^{\mathrm{dC}}$ \\
\hline & RGHAC (0.5) & $77.70 \pm 0.52^{\mathrm{aE}}$ & $73.35 \pm 0.32^{\mathrm{bE}}$ & $73.98 \pm 0.41^{\mathrm{cD}}$ & $72.68 \pm 0.5^{\mathrm{dE}}$ & $71.57 \pm 0.43^{\mathrm{eE}}$ \\
\hline \multirow[t]{7}{*}{$a^{*}$} & Control & $3.79 \pm 0.18^{\mathrm{cA}}$ & $3.78 \pm 0.18^{\mathrm{cA}}$ & $3.86 \pm 0.17^{\mathrm{cA}}$ & $7.93 \pm 0.16^{\mathrm{bA}}$ & $8.35 \pm 0.1^{\mathrm{aA}}$ \\
\hline & RGEAC (0.1) & $3.35 \pm 0.23^{\mathrm{dB}}$ & $3.30 \pm 0.09^{\mathrm{dB}}$ & $3.89 \pm 0.14^{\mathrm{cB}}$ & $7.93 \pm 0.2^{\mathrm{bA}}$ & $8.13 \pm 0.12^{\mathrm{aB}}$ \\
\hline & RGEAC (0.3) & $2.34 \pm 0.21^{\mathrm{eD}}$ & $2.84 \pm 0.2^{\mathrm{dD}}$ & $3.14 \pm 0.22^{\mathrm{cD}}$ & $7.07 \pm 0.27^{\mathrm{bC}}$ & $7.38 \pm 0.11^{\mathrm{aD}}$ \\
\hline & RGEAC (0.5) & $2.05 \pm 0.16^{\mathrm{dE}}$ & $2.45 \pm 0.11^{\mathrm{cE}}$ & $2.67 \pm 0.17^{\mathrm{bE}}$ & $6.86 \pm 0.32^{\mathrm{aD}}$ & $6.90 \pm 0.2^{\mathrm{aE}}$ \\
\hline & RGHAC (0.1) & $3.15 \pm 0.18^{\mathrm{cB}}$ & $3.36 \pm 0.19^{\mathrm{bB}}$ & $3.40 \pm 0.12^{\mathrm{bC}}$ & $7.77 \pm 0.12^{\mathrm{aA}}$ & $7.84 \pm 0.11^{\mathrm{aC}}$ \\
\hline & RGHAC (0.3) & $2.68 \pm 0.24^{\mathrm{dC}}$ & $3.03 \pm 0.27^{\mathrm{cC}}$ & $3.22 \pm 0.08^{\mathrm{bD}}$ & $7.33 \pm 0.1^{\mathrm{aD}}$ & $7.43 \pm 0.13^{\mathrm{aD}}$ \\
\hline & RGHAC (0.5) & $2.44 \pm 0.32^{\mathrm{dD}}$ & $2.68 \pm 0.17^{\mathrm{cD}}$ & $2.58 \pm 0.16^{\mathrm{cdE}}$ & $6.73 \pm 0.13^{\mathrm{bB}}$ & $7.01 \pm 0.31^{\mathrm{aE}}$ \\
\hline \multirow[t]{7}{*}{$b^{*}$} & Control & $10.35 \pm 0.88^{\mathrm{dE}}$ & $11.38 \pm 0.19^{\mathrm{cD}}$ & $11.64 \pm 0.38^{\mathrm{cD}}$ & $14.85 \pm 0.47^{\mathrm{bD}}$ & $15.56 \pm 0.16^{\mathrm{aD}}$ \\
\hline & RGEAC (0.1) & $11.46 \pm 0.26^{\mathrm{eCD}}$ & $11.81 \pm 0.31^{\mathrm{dC}}$ & $12.36 \pm 0.22^{\mathrm{cC}}$ & $15.77 \pm 0.52^{\mathrm{bC}}$ & $16.33 \pm 0.23^{\mathrm{aC}}$ \\
\hline & RGEAC (0.3) & $12.87 \pm 1.28^{\mathrm{bB}}$ & $13.10 \pm 0.52^{\mathrm{bB}}$ & $13.42 \pm 0.37^{\mathrm{bB}}$ & $17.36 \pm 0.19^{\mathrm{aB}}$ & $17.62 \pm 0.42^{\mathrm{aB}}$ \\
\hline & RGEAC (0.5) & $14.27 \pm 0.41^{\mathrm{dA}}$ & $14.54 \pm 0.25^{\mathrm{cA}}$ & $14.95 \pm 0.51^{\mathrm{cA}}$ & $17.99 \pm 0.44^{\mathrm{bA}}$ & $19.02 \pm 0.74^{\mathrm{aA}}$ \\
\hline & RGHAC (0.1) & $11.21 \pm 0.26^{\mathrm{eD}}$ & $11.79 \pm 0.35^{\mathrm{dC}}$ & $12.25 \pm 0.08^{\mathrm{cC}}$ & $15.60 \pm 0.47^{\mathrm{bC}}$ & $16.25 \pm 0.27^{\mathrm{aC}}$ \\
\hline & RGHAC (0.3) & $11.91 \pm 0.34^{\mathrm{dC}}$ & $13.24 \pm 0.37^{\mathrm{cB}}$ & $13.41 \pm 0.57^{\mathrm{cB}}$ & $17.08 \pm 0.46^{\mathrm{bB}}$ & $17.48 \pm 0.15^{\mathrm{aB}}$ \\
\hline & RGHAC (0.5) & $13.74 \pm 0.38^{\mathrm{dA}}$ & $14.23 \pm 0.69^{\mathrm{cA}}$ & $14.83 \pm 0.3^{\mathrm{bA}}$ & $17.86 \pm 0.32^{\mathrm{aA}}$ & $18.18 \pm 0.54^{\mathrm{aAB}}$ \\
\hline
\end{tabular}

Control, Asiago cheese; RGEAC, red ginseng extract-added Asiago cheese; RGHAC, red ginseng hydrolyzate-added Asiago cheese; SD, standard deviation.

${ }^{1} 0$ month means the sample obtained after 48 hours from cheese making

Values are mean $\pm \mathrm{SD}(\mathrm{n}=10)$. Means with different superscripts in a row $(\mathrm{a}-\mathrm{e})$ and column $(\mathrm{A}-\mathrm{G})$ are significant at $\mathrm{p}<0.05$ by Ducan's multiple range test.

$(\mathrm{p}<0.05)$. However, the $\mathrm{a}^{*}$ values significantly increased with an increasing ripening period, and increasing the concentrations of RGE and RGH $(0.1 \%$ to $0.5 \%$, w/w) in cheeses significantly decreased the $a^{*}$ value $(\mathrm{p}<0.05)$. The $\mathrm{b}^{*}$ values were also increased in the RGHAC and RGEAC as concentrations of $\mathrm{RGH}$ or $\operatorname{RGE}(0.1 \%$ to $0.5 \%$, wt/wt) were increased. According to Rohn and Jaros, (1996), a longer ripened time for hard cheeses resulted in a decrease of $\mathrm{L}^{*}$ value and an increase of $\mathrm{a}^{*}$ and $\mathrm{b}^{*}$ values. In the present study, a similar trend was observed in changes of color during the ripening periods due to the dark brown color of the RGE. These results are supported by the study of Kim et al. (2008) who also reported that the addition of RGE in yogurt greatly decreased $\mathrm{L}^{*}$ value and increased the $\mathrm{b}^{*}$ values with the increasing concentration of RGE.

\section{Texture profiles analysis}

The changes in the texture profile of RGHAC and RGEAC in terms of hardness, cohesiveness, gumminess, springiness, and chewiness during 4 months of ripening are shown in Table 5. Regarding hardness, all of the samples increased with increasing the ripening period, however, the concentrations of the RGH or RGE $(0.1 \%$ to $0.5 \%$, wt/wt) decreased the hardness of cheeses $(p<0.05)$. At 0 month ripening period, differences in hardness were markedly affected by adding RGH and RGE, but the hardness of RGHAC and RGEAC gradually became similar to that of the control during the ripening. In all samples, cohesiveness and springiness of control were ranged from 0.67 to 0.77 and from 0.82 to 0.93 , respectively, during ripening. However, the concentrations of RGH and RGE had no effect on the cohesiveness and springiness of cheese during ripening. Gumminess and chewiness were insignificantly increased with increasing the ripening time but the concentrations of RGH and RGE had no effect. This results can be explained by highly hydroscopic characteristics of red ginseng as shown in Table 1. Song et al. (2007) also reported that higher water absorption of RGE caused weak dough in white bread.

\section{Sensory evaluation}

The sensory attributes of the different concentrations $(0.1 \%, 0.3 \%$, and $0.5 \%$, wt/wt) of RGH-or RGE-added AC ripened at $14^{\circ} \mathrm{C}$ for 4 months are shown in Table 6 . At 0 month ripening, there was no significant difference in most sensory attributes of RGH and RGE in AC ( $p>0.05)$. However, ginseng flavor and taste were increased significantly with increasing the concentrations of RGH-or RGE-added to AC ( $\mathrm{p}<0.05)$. Increasing the RGH or RGE addition significantly increased yellowness score and 
Table 5. Texture properties of red ginseng hydrolyzate- or red ginseng extract-added Asiago cheeses ripened at $14^{\circ} \mathrm{C}$ for 4 months

\begin{tabular}{|c|c|c|c|c|c|c|}
\hline \multirow{2}{*}{$\begin{array}{l}\text { Texture } \\
\text { description }\end{array}$} & \multirow{2}{*}{$\begin{array}{c}\text { Concentration of } \\
\text { sample }(\%, \mathrm{wt} / \mathrm{wt})\end{array}$} & \multicolumn{5}{|c|}{ Ripening period (month) } \\
\hline & & $0^{1}$ & 1 & 2 & 3 & 4 \\
\hline \multirow{7}{*}{$\begin{array}{l}\text { Hardness } \\
\text { (N) }\end{array}$} & Control & $9.90 \pm 0.79^{\mathrm{cdA}}$ & $10.31 \pm 0.78^{\mathrm{cA}}$ & $10.29 \pm 1.52^{\mathrm{cA}}$ & $12.13 \pm 1.08^{\mathrm{bA}}$ & $15.19 \pm 1.06^{\mathrm{aA}}$ \\
\hline & RGEAC (0.1) & $7.94 \pm 0.83^{\mathrm{dB}}$ & $7.94 \pm 0.83^{\mathrm{dCD}}$ & $9.82 \pm 0.55^{\mathrm{cAB}}$ & $10.81 \pm 0.77^{\mathrm{bB}}$ & $13.09 \pm 0.48^{\mathrm{aB}}$ \\
\hline & RGEAC (0.3) & $7.23 \pm 0.43^{\mathrm{cBC}}$ & $8.78 \pm 0.84^{\mathrm{bB}}$ & $8.91 \pm 0.55^{\mathrm{bD}}$ & $11.39 \pm 0.96^{\mathrm{abAB}}$ & $12.62 \pm 0.56^{\mathrm{aBC}}$ \\
\hline & RGEAC (0.5) & $8.02 \pm 0.53^{\mathrm{cdB}}$ & $8.01 \pm 0.50^{\mathrm{cd}}$ & $9.06 \pm 0.62^{\mathrm{cC}}$ & $10.76 \pm 0.34^{\mathrm{bB}}$ & $12.70 \pm 1.00^{\mathrm{aB}}$ \\
\hline & RGHAC (0.1) & $7.00 \pm 1.64^{\mathrm{dC}}$ & $8.26 \pm 0.31^{\mathrm{cC}}$ & $9.38 \pm 1.31^{\mathrm{bcC}}$ & $10.90 \pm 0.74^{\mathrm{bB}}$ & $15.46 \pm 2.24^{\mathrm{aA}}$ \\
\hline & RGHAC (0.3) & $6.38 \pm 0.39^{\mathrm{dD}}$ & $7.77 \pm 0.64^{\mathrm{cD}}$ & $10.09 \pm 0.54^{\mathrm{bA}}$ & $10.85 \pm 0.66^{\mathrm{bB}}$ & $13.09 \pm 0.55^{\mathrm{aB}}$ \\
\hline & RGHAC (0.5) & $7.24 \pm 0.46^{\mathrm{dBC}}$ & $8.74 \pm 0.58^{\mathrm{cB}}$ & $9.92 \pm 0.80^{\mathrm{bAB}}$ & $9.87 \pm 0.42^{\mathrm{bC}}$ & $10.87 \pm 0.74^{\mathrm{aC}}$ \\
\hline \multirow[t]{7}{*}{ Cohesiveness } & Control & $0.77 \pm 0.01^{\mathrm{aA}}$ & $0.72 \pm 0.01^{\mathrm{bB}}$ & $0.69 \pm 0.02^{\mathrm{cD}}$ & $0.71 \pm 0.02^{\mathrm{bAB}}$ & $0.67 \pm 0.02^{\mathrm{dC}}$ \\
\hline & RGEAC (0.1) & $0.70 \pm 0.03^{\mathrm{aC}}$ & $0.70 \pm 0.03^{\mathrm{aBC}}$ & $0.71 \pm 0.02^{\mathrm{aC}}$ & $0.71 \pm 0.01^{\mathrm{aAB}}$ & $0.68 \pm 0.01^{\mathrm{bB}}$ \\
\hline & RGEAC (0.3) & $0.71 \pm 0.00^{\mathrm{bB}}$ & $0.73 \pm 0.02^{\mathrm{aB}}$ & $0.73 \pm 0.01^{\mathrm{aB}}$ & $0.69 \pm 0.02^{\mathrm{cC}}$ & $0.71 \pm 0.00^{\mathrm{bA}}$ \\
\hline & RGEAC (0.5) & $0.77 \pm 0.01^{\mathrm{aA}}$ & $0.71 \pm 0.01^{\mathrm{bB}}$ & $0.70 \pm 0.01^{\mathrm{bcCD}}$ & $0.72 \pm 0.02^{\mathrm{bA}}$ & $0.69 \pm 0.02^{\mathrm{cAB}}$ \\
\hline & RGHAC (0.1) & $0.72 \pm 0.01^{\mathrm{bB}}$ & $0.76 \pm 0.02^{\mathrm{aA}}$ & $0.71 \pm 0.00^{\mathrm{bC}}$ & $0.71 \pm 0.01^{\mathrm{bAB}}$ & $0.68 \pm 0.01^{\mathrm{cB}}$ \\
\hline & RGHAC (0.3) & $0.70 \pm 0.02^{\mathrm{bcC}}$ & $0.72 \pm 0.01^{\mathrm{bB}}$ & $0.76 \pm 0.03^{\mathrm{aA}}$ & $0.70 \pm 0.01^{\mathrm{bcB}}$ & $0.68 \pm 0.01^{\mathrm{cB}}$ \\
\hline & RGHAC (0.5) & $0.72 \pm 0.01^{\mathrm{bB}}$ & $0.69 \pm 0.03^{\mathrm{cC}}$ & $0.76 \pm 0.04^{\mathrm{aA}}$ & $0.70 \pm 0.01^{\mathrm{cB}}$ & $0.68 \pm 0.02^{\mathrm{cdB}}$ \\
\hline \multirow[t]{7}{*}{ Springiness } & Control & $0.82 \pm 0.11^{\mathrm{cD}}$ & $0.88 \pm 0.03^{\mathrm{bA}}$ & $0.82 \pm 0.00^{\mathrm{cC}}$ & $0.88 \pm 0.07^{\mathrm{bB}}$ & $0.93 \pm 0.09^{\mathrm{aA}}$ \\
\hline & RGEAC (0.1) & $0.87 \pm 0.08^{\mathrm{bB}}$ & $0.87 \pm 0.08^{\mathrm{bB}}$ & $0.82 \pm 0.01^{\mathrm{cC}}$ & $0.90 \pm 0.04^{\mathrm{aA}}$ & $0.89 \pm 0.09^{\mathrm{abBC}}$ \\
\hline & RGEAC (0.3) & $0.86 \pm 0.07^{\mathrm{bB}}$ & $0.88 \pm 0.12^{\mathrm{abA}}$ & $0.89 \pm 0.06^{\mathrm{abA}}$ & $0.88 \pm 0.03^{\mathrm{abB}}$ & $0.90 \pm 0.02^{\mathrm{aB}}$ \\
\hline & RGEAC (0.5) & $0.90 \pm 0.13^{\mathrm{aA}}$ & $0.89 \pm 0.01^{\mathrm{aA}}$ & $0.83 \pm 0.01^{\mathrm{cC}}$ & $0.85 \pm 0.06^{\mathrm{bC}}$ & $0.89 \pm 0.08^{\mathrm{aBC}}$ \\
\hline & RGHAC (0.1) & $0.84 \pm 0.01^{\mathrm{cBC}}$ & $0.83 \pm 0.08^{\mathrm{cB}}$ & $0.87 \pm 0.02^{\mathrm{bAB}}$ & $0.90 \pm 0.05^{\mathrm{aA}}$ & $0.88 \pm 0.08^{\mathrm{bC}}$ \\
\hline & RGHAC (0.3) & $0.84 \pm 0.02^{\mathrm{bBC}}$ & $0.90 \pm 0.03^{\mathrm{aA}}$ & $0.86 \pm 0.11^{\mathrm{bAB}}$ & $0.91 \pm 0.07^{\mathrm{aA}}$ & $0.92 \pm 0.07^{\mathrm{aAB}}$ \\
\hline & RGHAC (0.5) & $0.90 \pm 0.01^{\mathrm{aA}}$ & $0.86 \pm 0.02^{\mathrm{bAB}}$ & $0.85 \pm 0.09^{\mathrm{bB}}$ & $0.82 \pm 0.02^{\mathrm{cD}}$ & $0.92 \pm 0.06^{\mathrm{aB}}$ \\
\hline \multirow[t]{7}{*}{ Gumminess (N) } & Control & $7.64 \pm 0.66^{\mathrm{cA}}$ & $7.39 \pm 0.56^{\mathrm{cA}}$ & $7.07 \pm 0.87^{\mathrm{cB}}$ & $8.61 \pm 0.93^{\mathrm{bA}}$ & $10.43 \pm 1.45^{\mathrm{aA}}$ \\
\hline & RGEAC $(0.1)$ & $5.58 \pm 0.38^{\mathrm{dC}}$ & $5.58 \pm 0.38^{\mathrm{dC}}$ & $6.94 \pm 0.26^{\mathrm{cBC}}$ & $7.70 \pm 0.51^{\mathrm{bB}}$ & $8.85 \pm 0.46^{\mathrm{aB}}$ \\
\hline & RGEAC (0.3) & $5.14 \pm 0.29^{\mathrm{dCD}}$ & $6.48 \pm 0.51^{\mathrm{cB}}$ & $6.48 \pm 0.44^{\mathrm{cC}}$ & $7.85 \pm 0.78^{\mathrm{bB}}$ & $8.97 \pm 0.39^{\mathrm{aB}}$ \\
\hline & RGEAC (0.5) & $6.18 \pm 0.36^{\mathrm{cB}}$ & $5.73 \pm 0.38^{\mathrm{dC}}$ & $6.38 \pm 0.41^{\mathrm{cC}}$ & $7.77 \pm 0.36^{\mathrm{bB}}$ & $8.81 \pm 0.56^{\mathrm{aB}}$ \\
\hline & RGHAC (0.1) & $5.02 \pm 1.17^{\mathrm{dCD}}$ & $6.27 \pm 0.29^{\mathrm{cB}}$ & $6.64 \pm 0.95^{\mathrm{bcC}}$ & $6.96 \pm 0.33^{\mathrm{bD}}$ & $7.44 \pm 0.43^{\mathrm{aC}}$ \\
\hline & RGHAC (0.3) & $4.45 \pm 0.28^{\mathrm{dD}}$ & $5.60 \pm 0.48^{\mathrm{cC}}$ & $7.62 \pm 0.13^{\mathrm{bA}}$ & $7.57 \pm 0.46^{\mathrm{bBC}}$ & $10.38 \pm 0.77^{\mathrm{aA}}$ \\
\hline & RGHAC (0.5) & $5.23 \pm 0.31^{\mathrm{dC}}$ & $6.03 \pm 0.46^{\mathrm{cBC}}$ & $7.58 \pm 0.73^{\mathrm{bA}}$ & $7.66 \pm 0.36^{\mathrm{bC}}$ & $8.91 \pm 0.38^{\mathrm{aB}}$ \\
\hline \multirow[t]{7}{*}{ Chewiness } & Control & $6.25 \pm 0.80^{\mathrm{cdA}}$ & $6.50 \pm 0.51^{\mathrm{cA}}$ & $5.82 \pm 0.72^{\mathrm{dAB}}$ & $7.59 \pm 1.25^{\mathrm{bA}}$ & $9.74 \pm 1.76^{\mathrm{aA}}$ \\
\hline & RGEAC (0.1) & $4.82 \pm 0.37^{\mathrm{dBC}}$ & $4.82 \pm 0.37^{\mathrm{dD}}$ & $5.71 \pm 0.23^{\mathrm{cAB}}$ & $6.91 \pm 0.42^{\mathrm{bB}}$ & $7.86 \pm 1.10^{\mathrm{aB}}$ \\
\hline & RGEAC (0.3) & $4.44 \pm 0.48^{\mathrm{dC}}$ & $5.68 \pm 0.96^{\mathrm{cB}}$ & $5.77 \pm 0.43^{\mathrm{cAB}}$ & $7.69 \pm 0.78^{\mathrm{bA}}$ & $8.06 \pm 0.34^{\mathrm{aB}}$ \\
\hline & RGEAC (0.5) & $5.44 \pm 0.54^{\mathrm{cB}}$ & $5.11 \pm 0.30^{\mathrm{cdBC}}$ & $5.27 \pm 0.29^{\mathrm{cB}}$ & $6.63 \pm 0.70^{\mathrm{bBC}}$ & $7.84 \pm 1.12^{\mathrm{aB}}$ \\
\hline & RGHAC (0.1) & $4.22 \pm 1.05^{\mathrm{cCD}}$ & $5.21 \pm 0.57^{\mathrm{bcBC}}$ & $5.77 \pm 0.71^{\mathrm{bABB}}$ & $6.28 \pm 0.51^{\mathrm{ab}}$ & $6.58 \pm 0.91^{\mathrm{aC}}$ \\
\hline & RGHAC (0.3) & $3.76 \pm 0.23^{\mathrm{dD}}$ & $5.06 \pm 0.47^{\mathrm{cC}}$ & $6.54 \pm 0.96^{\mathrm{bA}}$ & $6.90 \pm 0.62^{\mathrm{bB}}$ & $9.56 \pm 0.47^{\mathrm{aA}}$ \\
\hline & RGHAC (0.5) & $4.71 \pm 0.27^{\mathrm{dBC}}$ & $5.10 \pm 0.44^{\mathrm{cBC}}$ & $6.41 \pm 0.32^{\mathrm{bA}}$ & $6.33 \pm 0.64^{\mathrm{bC}}$ & $8.22 \pm 0.56^{\mathrm{aB}}$ \\
\hline
\end{tabular}

Control, Asiago cheese; RGEAC, red ginseng extract-added Asiago cheese; RGHAC, red ginseng hydrolyzate-added Asiago cheese; SD, standard deviation.

${ }^{1} 0$ month means the sample obtained after 48 hours from cheese making.

Data values were expressed as mean $\pm \mathrm{SD}(\mathrm{n}=5)$. Means with different superscripts in a row (a-e) and column (A-G) are significant at $\mathrm{p}<0.05$ by Ducan's multiple range test.

cheese eye in 4 months $(\mathrm{p}<0.05)$. Larger number of cheese eye during 4 months could be attributed to the release of $\mathrm{CO}_{2}$ from the curd due to the relative softer texture. Flavor and taste properties, such as bitterness and astringency, were not significantly increased with increased of RGH and RGE ( $p>0.05)$. However, ginseng taste significantly increased with concentrations of RGE and RGH $(0.3 \%$ and $0.5 \%, \mathrm{wt} / \mathrm{wt}$ ). Increased concentrations of RGE and RGH at $0.5 \%(\mathrm{wt} / \mathrm{wt})$ significantly influenced the bitterness and astringency score AC during 4 month storage $(\mathrm{p}<0.05)$.
Hardness score was lowered with additions of RGH and RGE to AC in early ripening period, however, there was little effect after 4 month storage. Most studies reported that additions of the red ginseng to other foods, such as Topokki, Garaedduck, Yackwa, Tofu etc. showed negative effects on preference (Hyun and Kim, 2005; Lee et al., 2008; Lee et al., 2011). However, ginseng hydrolyzates and extracts revealed positive results in $0.1 \%$ and $0.3 \%$ added to chesses during ripening. 
Table 6. Sensory evaluation of the red ginseng hydrolyzate- or red ginseng extracts-added Asiago cheese ripened at $14^{\circ} \mathrm{C}$ for 4 months

\begin{tabular}{|c|c|c|c|c|c|c|}
\hline \multirow{2}{*}{$\begin{array}{l}\text { Concentration } \\
\text { of sample }(\%, w t / w t)\end{array}$} & \multicolumn{2}{|c|}{ Appearance } & \multicolumn{3}{|c|}{ Flavor and taste } & \multirow{2}{*}{$\begin{array}{c}\text { Texture } \\
\text { Hardness }\end{array}$} \\
\hline & Yellowness & Cheese eye & Bitterness & Astringency & Ginseng & \\
\hline \multicolumn{7}{|l|}{0 -m ripening period ${ }^{1}$} \\
\hline Control & $4.00 \pm 0.00^{\mathrm{a}}$ & $4.00 \pm 0.00^{\mathrm{a}}$ & $4.00 \pm 0.00^{\mathrm{a}}$ & $4.00 \pm 0.00^{\mathrm{a}}$ & $1.00 \pm 0.00^{c}$ & $4.00 \pm 0.00^{\mathrm{a}}$ \\
\hline RGEAC (0.1) & $4.57 \pm 0.38^{\mathrm{a}}$ & $4.00 \pm 0.00^{\mathrm{a}}$ & $3.86 \pm 0.38^{\mathrm{a}}$ & $4.14 \pm 1.22^{\mathrm{a}}$ & $1.57 \pm 0.79^{\mathrm{bc}}$ & $4.14 \pm 0.69^{\mathrm{a}}$ \\
\hline RGEAC (0.3) & $4.57 \pm 0.54^{\mathrm{a}}$ & $4.29 \pm 0.49^{\mathrm{a}}$ & $4.14 \pm 0.90^{\mathrm{a}}$ & $4.71 \pm 0.76^{\mathrm{a}}$ & $1.57 \pm 0.79^{\mathrm{bc}}$ & $4.00 \pm 0.58^{\mathrm{a}}$ \\
\hline RGEAC (0.5) & $4.57 \pm 0.79^{\mathrm{a}}$ & $4.14 \pm 0.38^{\mathrm{a}}$ & $4.14 \pm 0.90^{\mathrm{a}}$ & $4.29 \pm 0.76^{\mathrm{a}}$ & $2.71 \pm 1.11^{\mathrm{a}}$ & $4.00 \pm 0.58^{\mathrm{a}}$ \\
\hline RGHAC (0.1) & $4.00 \pm 0.0^{\mathrm{a}}$ & $4.14 \pm 0.38^{\mathrm{a}}$ & $3.57 \pm 0.89^{\mathrm{a}}$ & $4.00 \pm 0.00^{\mathrm{a}}$ & $1.14 \pm 0.38^{\mathrm{c}}$ & $4.00 \pm 0.39^{\mathrm{a}}$ \\
\hline RGHAC (0.3) & $4.14 \pm 0.38^{\mathrm{a}}$ & $4.14 \pm 0.38^{\mathrm{a}}$ & $4.00 \pm 0.58^{\mathrm{a}}$ & $4.14 \pm 0.69^{\mathrm{a}}$ & $1.57 \pm 0.54^{\mathrm{bc}}$ & $3.85 \pm 0.69^{\mathrm{a}}$ \\
\hline RGHAC (0.5) & $4.14 \pm 0.79^{\mathrm{a}}$ & $4.29 \pm 0.49^{\mathrm{a}}$ & $4.00 \pm 0.58^{\mathrm{a}}$ & $3.86 \pm 0.69^{\mathrm{a}}$ & $2.29 \pm 1.38^{\mathrm{ab}}$ & $3.85 \pm 0.00^{\mathrm{a}}$ \\
\hline \multicolumn{7}{|l|}{ 1-m ripening period } \\
\hline Control & $4.00 \pm 0.00^{\mathrm{c}}$ & $4.00 \pm 0.00^{\mathrm{a}}$ & $4.00 \pm 0.00^{\mathrm{c}}$ & $4.00 \pm 0.00^{\mathrm{a}}$ & $1.00 \pm 0.00^{\mathrm{c}}$ & $4.00 \pm 0.00^{\mathrm{a}}$ \\
\hline RGEAC (0.1) & $4.14 \pm 0.38^{\mathrm{c}}$ & $4.00 \pm 0.58^{\mathrm{a}}$ & $4.29 \pm 0.76^{\mathrm{c}}$ & $4.00 \pm 0.82^{\mathrm{a}}$ & $1.71 \pm 0.49^{\mathrm{bc}}$ & $3.86 \pm 0.69^{\mathrm{a}}$ \\
\hline RGEAC (0.3) & $4.86 \pm 0.38^{\mathrm{b}}$ & $4.14 \pm 0.38^{\mathrm{a}}$ & $4.58 \pm 0.79^{\mathrm{abc}}$ & $4.43 \pm 0.98^{\mathrm{a}}$ & $2.86 \pm 1.35^{\mathrm{ab}}$ & $3.58 \pm 0.54^{\mathrm{a}}$ \\
\hline RGEAC (0.5) & $5.57 \pm 0.54^{\mathrm{a}}$ & $4.42 \pm 0.79^{\mathrm{a}}$ & $4.71 \pm 0.76^{\mathrm{ab}}$ & $4.43 \pm 0.79^{\mathrm{a}}$ & $3.86 \pm 1.35^{\mathrm{a}}$ & $4.00 \pm 0.82^{\mathrm{a}}$ \\
\hline RGHAC (0.1) & $4.00 \pm 0.58^{\mathrm{c}}$ & $4.14 \pm 0.38^{\mathrm{a}}$ & $4.71 \pm 1.11^{\mathrm{abc}}$ & $4.00 \pm 0.82^{\mathrm{a}}$ & $1.43 \pm 0.54^{\mathrm{c}}$ & $3.29 \pm 0.49^{\mathrm{a}}$ \\
\hline RGHAC (0.3) & $5.00 \pm 0.58^{\mathrm{b}}$ & $4.00 \pm 0.58^{\mathrm{a}}$ & $5.00 \pm 0.58^{\mathrm{ab}}$ & $4.71 \pm 0.76^{\mathrm{a}}$ & $2.71 \pm 0.95^{\mathrm{ab}}$ & $3.29 \pm 0.49^{\mathrm{a}}$ \\
\hline RGHAC (0.5) & $5.71 \pm 0.76^{\mathrm{a}}$ & $4.29 \pm 0.49^{\mathrm{a}}$ & $5.14 \pm 0.90^{\mathrm{ab}}$ & $4.86 \pm 1.22^{\mathrm{a}}$ & $3.00 \pm 1.53^{\mathrm{a}}$ & $3.58 \pm 0.79^{\mathrm{a}}$ \\
\hline \multicolumn{7}{|l|}{ 2-m ripening period } \\
\hline Control & $4.00 \pm 0.00^{c}$ & $4.00 \pm 0.00^{\mathrm{a}}$ & $4.00 \pm 0.00^{c}$ & $4.00 \pm 0.00^{\mathrm{a}}$ & $1.00 \pm 0.00^{\mathrm{c}}$ & $4.00 \pm 0.00^{\mathrm{a}}$ \\
\hline RGEAC (0.1) & $4.14 \pm 0.38^{\mathrm{c}}$ & $4.00 \pm 0.82^{\mathrm{a}}$ & $4.29 \pm 0.76^{\mathrm{bc}}$ & $3.86 \pm 1.07^{\mathrm{a}}$ & $1.71 \pm 0.49^{\mathrm{bc}}$ & $3.86 \pm 0.69^{\mathrm{ab}}$ \\
\hline RGEAC (0.3) & $4.86 \pm 0.38^{\mathrm{b}}$ & $4.14 \pm 0.38^{\mathrm{a}}$ & $4.57 \pm 0.79^{\mathrm{abc}}$ & $4.29 \pm 1.25^{\mathrm{a}}$ & $2.86 \pm 1.35^{\mathrm{ab}}$ & $3.86 \pm 1.07^{\mathrm{ab}}$ \\
\hline RGEAC (0.5) & $5.57 \pm 0.54^{\mathrm{a}}$ & $4.57 \pm 0.98^{\mathrm{a}}$ & $5.29 \pm 0.76^{\mathrm{a}}$ & $4.43 \pm 0.79^{\mathrm{a}}$ & $3.86 \pm 1.35^{\mathrm{a}}$ & $3.43 \pm 0.79^{\mathrm{ab}}$ \\
\hline RGHAC (0.1) & $4.00 \pm 0.58^{\mathrm{c}}$ & $4.14 \pm 0.69^{\mathrm{a}}$ & $4.71 \pm 1.11^{\mathrm{abc}}$ & $3.86 \pm 1.07^{\mathrm{a}}$ & $1.43 \pm 0.54^{\mathrm{c}}$ & $3.29 \pm 0.49^{\mathrm{ab}}$ \\
\hline RGHAC (0.3) & $5.00 \pm 0.58^{\mathrm{b}}$ & $4.57 \pm 0.98^{\mathrm{a}}$ & $5.00 \pm 0.58^{\mathrm{ab}}$ & $5.00 \pm 0.82^{\mathrm{a}}$ & $2.71 \pm 0.95^{\mathrm{ab}}$ & $3.57 \pm 0.79^{\mathrm{ab}}$ \\
\hline RGHAC (0.5) & $5.71 \pm 0.76^{\mathrm{a}}$ & $4.86 \pm 1.07^{\mathrm{a}}$ & $5.14 \pm 0.90^{\mathrm{ab}}$ & $4.571 .13^{\mathrm{a}}$ & $3.00 \pm 1.53^{\mathrm{a}}$ & $3.00 \pm 0.82^{\mathrm{b}}$ \\
\hline \multicolumn{7}{|l|}{$3-\mathrm{m}$ ripening period } \\
\hline Control & $4.00 \pm 0.00^{c}$ & $4.00 \pm 0.00^{\mathrm{a}}$ & $4.00 \pm 0.00^{\mathrm{a}}$ & $4.00 \pm 0.00^{\mathrm{a}}$ & $1.00 \pm 0.00^{\mathrm{c}}$ & $4.00 \pm 0.00^{\mathrm{b}}$ \\
\hline RGEAC (0.1) & $4.57 \pm 0.98^{\mathrm{bc}}$ & $4.29 \pm 0.49^{\mathrm{a}}$ & $4.00 \pm 0.82^{\mathrm{a}}$ & $4.14 \pm 1.07^{\mathrm{a}}$ & $2.14 \pm 0.38^{\mathrm{b}}$ & $4.43 \pm 0.54^{\mathrm{ab}}$ \\
\hline RGEAC (0.3) & $5.14 \pm 0.38^{\mathrm{b}}$ & $4.43 \pm 0.54^{\mathrm{a}}$ & $4.43 \pm 0.79^{\mathrm{a}}$ & $4.14 \pm 0.69^{\mathrm{a}}$ & $2.43 \pm 0.79^{\mathrm{ab}}$ & $4.43 \pm 0.54^{\mathrm{ab}}$ \\
\hline RGEAC (0.5) & $5.86 \pm 0.69^{\mathrm{a}}$ & $4.57 \pm 0.98^{\mathrm{a}}$ & $4.43 \pm 0.98^{\mathrm{a}}$ & $5.00 \pm 0.82^{\mathrm{a}}$ & $3.00 \pm 0.82^{\mathrm{a}}$ & $4.57 \pm 1.13^{\mathrm{ab}}$ \\
\hline RGHAC (0.1) & $4.43 \pm 0.54^{c}$ & $4.00 \pm 0.82^{\mathrm{a}}$ & $4.43 \pm 1.27^{\mathrm{a}}$ & $4.71 \pm 1.11^{\mathrm{a}}$ & $1.29 \pm 0.49^{c}$ & $4.71 \pm 0.95^{\mathrm{ab}}$ \\
\hline RGHAC (0.3) & $5.14 \pm 0.38^{\mathrm{b}}$ & $4.00 \pm 0.90^{\mathrm{a}}$ & $4.00 \pm 0.82^{\mathrm{a}}$ & $4.14 \pm 0.69^{\mathrm{a}}$ & $2.14 \pm 1.07^{\mathrm{b}}$ & $3.00 \pm 0.82^{\mathrm{a}}$ \\
\hline RGHAC (0.5) & $6.00 \pm 0.58^{\mathrm{a}}$ & $4.29 \pm 0.49^{\mathrm{a}}$ & $4.43 \pm 0.54^{\mathrm{a}}$ & $4.43 \pm 0.79^{\mathrm{a}}$ & $2.43 \pm 0.54^{\mathrm{ab}}$ & $3.00 \pm 0.82^{\mathrm{a}}$ \\
\hline \multicolumn{7}{|l|}{ 4-m ripening period } \\
\hline Control & $4.00 \pm 0.00^{\mathrm{b}}$ & $4.00 \pm 0.00^{\mathrm{c}}$ & $4.00 \pm 0.00^{c}$ & $4.00 \pm 0.00^{\mathrm{b}}$ & $1.00 \pm 0.00^{\mathrm{c}}$ & $4.00 \pm 0.00^{\mathrm{ab}}$ \\
\hline RGEAC (0.1) & $4.00 \pm 0.58^{\mathrm{b}}$ & $4.86 \pm 0.90^{\mathrm{b}}$ & $4.29 \pm 0.49^{\mathrm{bc}}$ & $4.43 \pm 0.79^{b}$ & $2.29 \pm 1.25^{\mathrm{b}}$ & $3.57 \pm 0.54^{\mathrm{b}}$ \\
\hline RGEAC (0.3) & $5.29 \pm 0.49^{\mathrm{a}}$ & $4.86 \pm 0.38^{b}$ & $4.57 \pm 0.98^{\mathrm{bc}}$ & $4.43 \pm 0.54^{b}$ & $3.14 \pm 1.35^{\mathrm{ab}}$ & $4.29 \pm 1.11^{\mathrm{ab}}$ \\
\hline RGEAC (0.5) & $5.57 \pm 0.79^{\mathrm{a}}$ & $5.43 \pm 0.54^{\mathrm{ab}}$ & $5.71 \pm 0.95^{\mathrm{a}}$ & $5.86 \pm 0.90^{\mathrm{sb}}$ & $3.29 \pm 0.76^{\mathrm{ab}}$ & $4.43 \pm 0.98^{\mathrm{ab}}$ \\
\hline RGHAC (0.1) & $4.00 \pm 0.58^{\mathrm{b}}$ & $5.71 \pm 0.49^{\mathrm{a}}$ & $4.43 \pm 0.98^{\mathrm{bc}}$ & $4.86 \pm 1.35^{\mathrm{b}}$ & $2.29 \pm 0.49^{\mathrm{b}}$ & $3.86 \pm 0.90^{\mathrm{b}}$ \\
\hline RGHAC (0.3) & $5.29 \pm 0.95^{\mathrm{a}}$ & $5.43 \pm 0.79^{\mathrm{ab}}$ & $4.71 \pm 0.76^{\mathrm{bc}}$ & $4.71 \pm 0.49^{\mathrm{b}}$ & $2.71 \pm 0.76^{\mathrm{ab}}$ & $3.57 \pm 0.79^{b}$ \\
\hline RGHAC (0.5) & $5.43 \pm 0.96^{\mathrm{a}}$ & $6.00 \pm 0.58^{\mathrm{a}}$ & $5.14 \pm 1.35^{\mathrm{ab}}$ & $5.00 \pm 1.16^{\mathrm{ab}}$ & $3.86 \pm 1.57^{\mathrm{a}}$ & $5.00 \pm 1.16^{\mathrm{a}}$ \\
\hline
\end{tabular}

Control, Asiago cheese; RGEAC, red ginseng extract-added Asiago cheese; RGHAC, red ginseng hydrolyzate-added Asiago cheese; SD, standard deviation.

${ }^{1} 0$ month means the sample obtained after 48 hours from cheese making

Data values were expressed as mean $\pm \mathrm{SD}(\mathrm{n}=7)$. Means with different superscripts in a row $(\mathrm{a}-\mathrm{g})$ is significant at $\mathrm{p}<0.05$ by Ducan's multiple range test.

\section{CONCLUSION}

The current study was designed to develop RGH- or RGE-added AC and to evaluate the effect of adding RGH and RGE on the physicochemical, texture, and sensory properties during ripening. The data on the $\mathrm{LAB}$, color, texture, and sensory analysis obtained from the current study indicated that concentrations ( $0.1 \%$ to $0.3 \%)$ of RGH and RGE could be applicable in RGH- or RGE-added cheese development. The production of AC that incorporates RGH or RGE can broaden the utilization of red ginseng, and the products can be regarded as possible health-promoting functional foods. 


\section{ACKNOWLEDGMENTS}

The present study was supported by a grant from Korea Institute of Planning and Evaluation for Technology in Food, Agriculture, Forestry and Fisheries in Gyeonggi-Do, Republic of Korea.

\section{REFERENCES}

Akao, T., M. Kanaoka, and K. Kobashi. 1998. Appearance of compound $\mathrm{K}$, a major metabolite of ginsenoside $\mathrm{Rb} 1$ by intestinal bacteria, in rat plasma after oral administration measurement of compound $\mathrm{K}$ by enzyme immunoassay. Biol. Pharm. Bull. 21:245-249.

AOAC International. 2000. Official Methods of Analysis. Vol. I. 19th ed. Association of Official Analytical Chemists International, Washington DC, USA.

Bae, E. A., M. J. Han, M. K. Choo, S. Y. Park, and D. H. Kim. 2002. Metabolism of 20(S)- and 20(R)-ginsenoside Rg3 by human intestinal bacteria and its relation to in vitro biological activities. Biol. Pharm. Bull. 25:58-63.

Chung, S. H., C. G. Choi, and S. H. Park. 2001. Comparisons between white ginseng radix and rootlet for antidiabetic activity and mechanism in KKAy mice. Arch. Pharm. Res. 24: 214-218.

Cochran, W. G. and G. M. Cox. 1957. Experimental Designs. John WILEY \& Sons, New York, NY, USA.

Gazzetta, Ufficiale. 2006. Regulation of cheese production DOP "Asiago". Gazzetta Ufficialedella Repubblica Italiana n. 190 of 17/08/2006

Hyun, J. S. and M. A. Kim. 2005. The effect of addition of level of red ginseng powder on Yackwa quality and during storage. Korean J. Food Cult. 20:352-359.

Jung, H. J., P. Ganesan, S. J. Lee, and H. S. Kwak. 2013. Comparative study of flavor in cholesterol-removed Gouda cheese and Gouda cheese during ripening. J. Dairy Sci. 96:1972-1983.

Kanawjia, S. K., P. Rajesh, L. Sabikhi, and S. Singh. 1995. Flavour, chemical and textural profile changes in accelerated ripened Gouda cheese. Lebensmittel-Wissenschaft und Technology 22:248-254.
Kim, J. H., D. H. Hahm, D. C. Yang, J. H. Kim, H. J. Lee, and I. Shim. 2005. Effect of crude saponin of Korean red ginseng on high-fat diet-induced obesity in the rat. J. Pharm. Sci. 97:124131.

Kim, J. J., S. H. Yu, W. M. Jeon, and H. S. Kwak. 2006. The effect of evening primrose oil on chemical and blood cholesterol lowering properties of Cheddar cheese. Asian Australas. J. Anim. Sci. 19:450-458.

Kim, S. I., S. H. Ko, Y. J. Lee, H. Y. Choi, and Y. S. Han. 2008. Antioxidant activity of yogurt supplemented with red ginseng extract. Korean J. Food Cookery Sci. 24:358-366

Kwon, S. W., S. B. Han, I. H. Park, J. M. Kim, M. K. Park, and J. H. Park. 2001. Liquid chromatographic determination of less polar ginsenosides in processed ginseng. J. Chromatogr. A 921:335-339.

Lee, J. K., J. H. Jeong, and J. K. Lim. 2011. Quality characteristics of Topokki Garaedduk added with ginseng powder. J. Korean Soc. Food Sci. Nutr. 40:426-434.

Lee, N. H., S. R. Yoo, H.G. Kim, J. H. Cho, and C. G. Son. 2012. Safety and tolerability of Panax ginseng root extract: a randomized, placebo-controlled, clinical trial in healthy Korean volunteers. J. Altern. Complement. Med. 18:10611069.

Lee, Y. S., I. S. Chung, I. R. Lee, K. H. Kim, W. S. Hong, and Y. S. Yun. 1997. Activation of multiple effector pathways of immune system by the antineoplastic immunostimulator acidic polysaccharide ginsan isolated from Panax ginseng. Anticancer. Res. 17:323-331.

Lee, J. S., G. N. Kim, and H. D. Jang. 2008. Effect of red ginseng extract on storage and antioxidant activity of tofu. J. Korean Soc. Food Sci. Nutr. 37:1497-1506.

Paek, I. B., Y. Moon, J. Kim, H. Y. Ji, S. A. Kim, D. H. Sohn, J. B. Kim, and H. S. Lee. 2006. Pharmacokinetics of a ginseng saponin metabolite compound $\mathrm{K}$ in rats. Biopharm. Drug Dispos. 27:39-45.

Rohm, H. and Jaros D. 1996. Colour of hard cheeses 1. Description of coulor properties and effects of maturation. $\mathrm{Z}$ Lebensm. Unters. Fosch. 203:241-244.

Song, M. R., K. S. Lee, B. C. Lee, and M. J. Oh. 2007. Quality and sensory characteristics of white breads added with various ginseng products. Korean J. Food Presrev. 14:369-377. 